Article

\title{
Analysis of the contribution of water vapor from the Arabian Sea to the local rainstorm process in southern Xinjiang
}

\author{
Hua Gao ${ }^{1,2}$, Dongling Bao ${ }^{3, *}$,Zuofang Zheng ${ }^{2}$,and Mamatabdulla Emer ${ }^{1,4}$ \\ 1 Institute of Desert Meteorology, China Meteorological Administration, Urumqi 830002, China; \\ hgao@ium.cn (H.G.); htmmt@163.com (M.E.) \\ 2 Institute of Urban Meteorology, China Meteorological Administration, Beijing 100089, China; zfzheng@ium.cn (Z.Z);
}

393110 troops of the Chinese People's Liberation Army, Beijing, 100089, China; bd10124@ sina.cn (D.B.)

4 Hotan Meteorological Bureau, Hotan 848000, Xinjiang, China; htmmt@163.com (M.E.)

* Correspondence: bd10124@ sina.com(D.B); Tel.: +86-13520649773*(D.B)

\begin{abstract}
Based on NCEP FNL $1^{\circ *} 1^{\circ}$ analysis data and Xinjiang meteorological bureau area numerical operation prediction, a local precipitation event in southern Xinjiang on June 26 was analyzed, and the results showed that the water vapor involved in this process originated from the northwestern Gangetic Plain and was transported along the Indus River plain and over the northwestern end of the mountains. Driven by the Iranian low-pressure trough, water vapor at 500 $\mathrm{hPa}$ was transported across the northwestern end of the Gangdise Mountains, past Georgoli Peak, and northwest over the Kunlun Mountains to arrive in southern Xinjiang.
\end{abstract}

Keywords: water vapor; local rainstorm; southern Xinjiang; Arabian Sea.

\section{Introduction}

Due to the complexity of multiscale physical processes and the local environment, local precipitation forecasting has long been a subject of interest and difficulty in scientific research and business. The regional numerical forecast system (RMAPS-CAv1.0) localized by the Xinjiang meteorological bureau based on the WRF-ARW (Weather Research and Forecasting - Advanced Research) Version 3.8.1 model has the advantages of high spatial and temporal resolution and plays an indispensable reference role in province-level forecasting. With the improvement of the spatial and temporal resolution of the regional numerical model, multiscale analysis of the local precipitation process has become possible. Zhang[1] analyzed the precipitation area and intensity of two rare rainstorms in southern Xinjiang in 2013 and found that there were three main transport routes for water vapor: west, north and east. Shi[2-3] analyzed the climatic characteristics and areal rainfall distribution of water vapor transport in Xinjiang. Zeng[4-8] analyzed the track of water vapor transport during a heavy rainstorm in western Xinjiang and analyzed the differences in water vapor contribution among the main channels and different sources. Li[9-12] analyzed the role of the weather scale and geographical conditions of heavy rainfall in Xinjiang. Wang[13] observed and analyzed the tropospheric height and water vapor transport in summer in the Minfeng region, southern Xinjiang. 
The results show that the water vapor over the northwestern Qinghai-Tibet Plateau is transported to the southern margin of the Tarim Basin under the influence of westerly and southwesterly winds at the base of the subtropical westerly jet stream. Other studies by previous researchers[14-16] have also identified different vapor sources in southern Xinjiang. However, the source channel of water vapor in the northwest of the Qinghai-Tibet Plateau has not been discussed. The southern part of Xinjiang is located in the leeward zone of the Qinghai-Tibet Plateau. While there is little rain year round and the average annual precipitation is less than $100 \mathrm{~mm}$, the precipitation process on June 26, 2019, was characterized by a high intensity, concentrated precipitation area and other medium- and small-scale features, which are rare in southern Xinjiang. This study mainly analyzes the source of the water vapor and the transport channel associated with this local heavy rainfall process.

\section{Actual precipitation}

From 00:00 to 24:00 on June 26, 2019 (UTC), rare local heavy precipitation occurred in the southern part of Xinjiang. The total precipitation within 24 hours reached $48.8 \mathrm{~mm}$ (Figure 1), which corresponds to the level of heavy rain based on the precipitation level standard set by Xinjiang in 2004. This precipitation event was characterized by a high degree of localization and a high intensity. The cumulative precipitation was the second highest in the history of the meteorological station.

The circulation pattern of the $500 \mathrm{hPa}$ low-pressure trough in western Siberia and the Afghan low-pressure vortex provided a favorable circulation situation for the occurrence and development of the rainstorm. In particular, the Afghan vortex provided the dynamic conditions for moisture from the Arabian Sea to cross the Qinghai-Tibet Plateau and enter the southern margin of the Tarim Basin through the Indus Plain. 


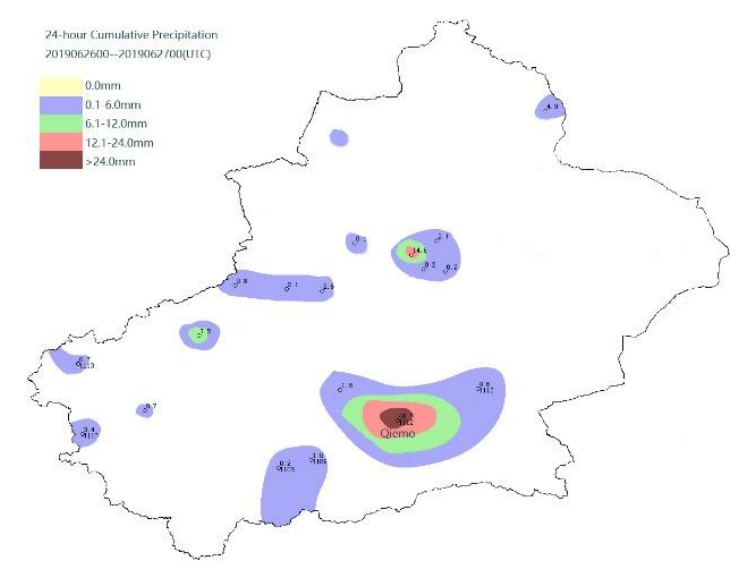

Figure 1 Twenty-four-hour cumulative precipitation observations

\section{Data}

Based on FNL $1^{\circ} * 1^{\circ}$ analysis data and RMAPS-CAv1.0[17] prediction results, the water vapor source in the precipitation process was analyzed. RMAPS-CAv1.0 is a regional numerical prediction system based on the WRFv3.8.1 prediction model and WRFDA assimilation platform. The system is two-fold nested (Figure 2), with an outer zone with a resolution of $9 \mathrm{~km}$ covering central Asia, and an inner zone with a resolution of $3 \mathrm{~km}$ covering Xinjiang, Gansu and western Qinghai. The system adopts the Global Forecast System (GFS) prediction field at the 6th hour as the background field, the initial field in the inner region is obtained by the ndown method in the outer region, and the 48-hour prediction is carried out after the assimilation of both regions. The assimilated data include microroutine observations and local special observations, including ground observations, sounding observations, aviation reports, etc. The parameterized configuration is shown in Table 1. 


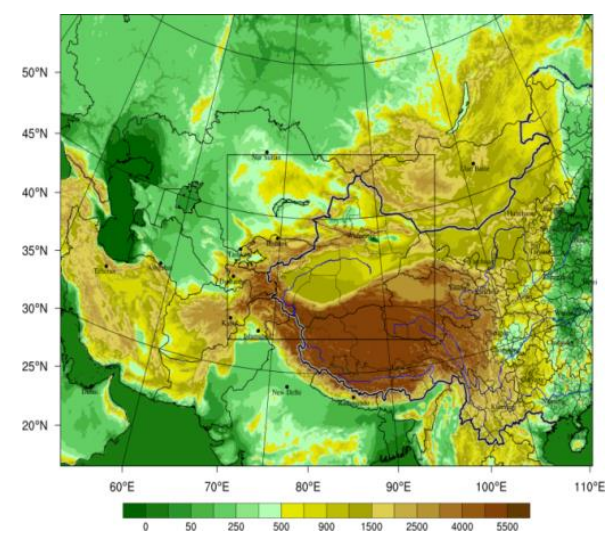

Figure 2 Two nested domains and topography

Table 1 Model parameter configuration

\begin{tabular}{ll}
\hline The name of the system & RMAPS - CAv1.0 \\
Schema versions & WPSV3.8.1 + WRFDAV3.8.1 + WRFV3.8.1 \\
Model range & $9 \mathrm{~km}: 712 * 532 * 50$ \\
And the resolution & $3 \mathrm{~km}: 832 * 652 * 50$ \\
Run the framework & 00, 12 UTC adopts cold start, then hot start for 6 hours, \\
The background field $9 / 3 \mathrm{~km}$ cycle update forecast is performed & NCEP GFS 0.5 degree forecast \\
& Three-dimensional variational assimilation including: \\
- & General GTS data \\
Data assimilation & Xinjiang automatic station data \\
& Radial wind observation by 11 radars in Xinjiang \\
PBL: ACM2 SW: RRTMG \\
LW: RRTMG LSM: NOAH \\
MP: WSM6
\end{tabular}

\section{Moisture analysis}

\subsection{Water vapor height}

Since the average elevation of the Qinghai-Tibet Plateau is above 5500 meters, the distribution of water vapor only above $500 \mathrm{hPa}$ is considered in this study due to the topography. As shown in Figure 3, from the height above the $0^{\circ}$ line to the height of $10 \mathrm{~km}$, 
there are high humidity zones with relative humidity values above $80 \%$ over a long distance. This situation provided a source of water vapor for this event.

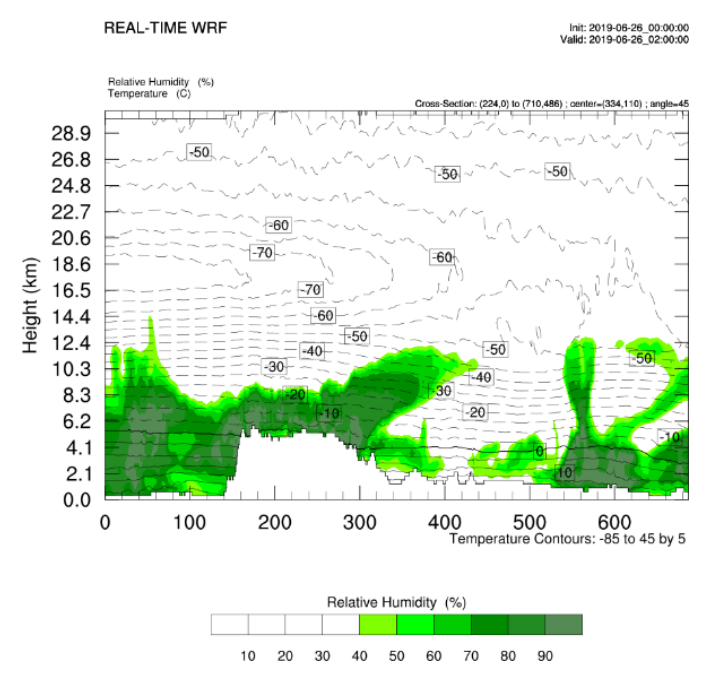

Figure 3 Profile of the humidity field

\subsection{Water vapor channel}

In the 24 hours before the event, the combined impact of the low-pressure vortex over central Asia and cyclone over Bangladesh allowed water vapor to be transported from the Arabian Sea over the plains of the Indus River, across the mountains to the northwest and the Kunlun Mountains to the north at heights above $500 \mathrm{hPa}$. This formed a stable moisture channel from the southwest to the northeast, and large amounts of water vapor were transported to southern Xinjiang (Figure 4).

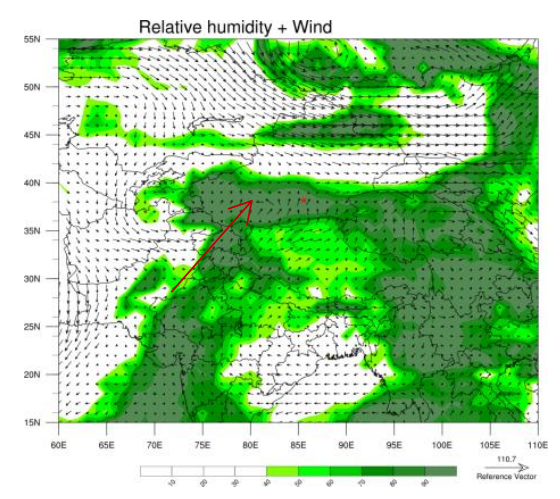




\subsection{Water vapor field}

One of the necessary conditions for a rainstorm is an adequate supply of water vapor. In the 24 hours preceding the precipitation event, due to the combined effects of the northern cyclone in the Bay of Bengal and the Afghan low-pressure vortex, there was a continuous strong southwest wind along the Indus Plain from the Qinghai-Tibet Plateau to the Tarim Basin. The vertical profile showed that there was a continuous high humidity zone with relative humidity greater than $90 \%$ between altitudes of $6 \mathrm{~km}$ and $8 \mathrm{~km}$. From the section analysis of the southern margin of the Tarim Basin, water vapor was continuously transported from south to north over time.

The formula for calculating the water vapor flux into southern Xinjiang during this process is:

$$
\begin{aligned}
& M_{v}= \\
& \frac{1}{g}|V| q \Delta \mathrm{l}|\Delta p|
\end{aligned}
$$

The formula for calculating the amount of water vapor flowing into southern Xinjiang during this process is as follows:

$$
M=
$$

$$
\iint M_{v} d x d y
$$

In the above equation, each physical quantity is as follows:

$|V|$ denotes the magnitude of the wind speed; $\mathrm{q}$ is the specific humidity; $\Delta \mathrm{l}$ is the unit length; and $\Delta p$ is unit air column pressure. The units of these parameters are as follows:

$\mathrm{g} \sim \mathrm{m} \cdot s^{-2},|V| \sim \mathrm{m} \cdot s^{-1}, \quad \mathrm{q} \sim \mathrm{g} \cdot \mathrm{kg}^{-1}, \quad \Delta \mathrm{l} \sim \mathrm{m}, \quad \Delta p \sim \mathrm{hPa}$ 
A zonal profile was made along the northern foothills of the Kunlun Mountains (latitude $36^{\circ} \mathrm{N}$ ), and the width was limited to the longitude range $\left[70^{\circ} \mathrm{E}, 80^{\circ} \mathrm{E}\right]$ and the height range [500 $\mathrm{hPa}, 300 \mathrm{hPa}$ ] according to the water vapor channel scope of the process described in section 4.2. Then, formula (2) is used to calculate the quantity of water vapor reaching the southern region of Xinjiang after passing through the section, as shown in Figure 5.

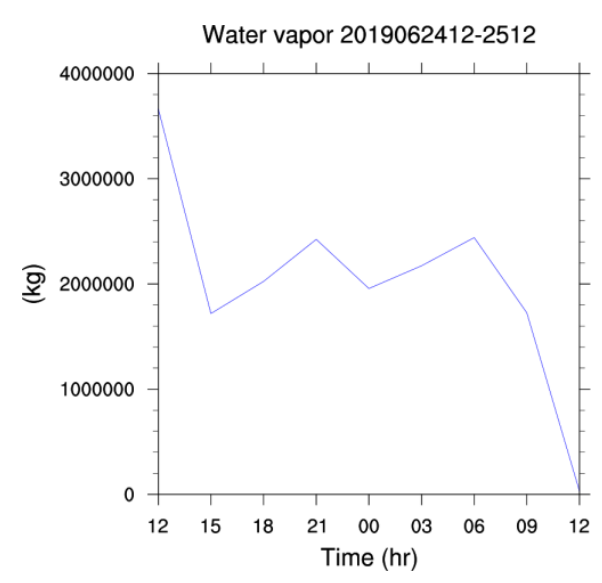

Figure 5 Curve of water vapor delivery with time

In the first 24 hours after the end of the precipitation process, the total water vapor input and transmission amount was more than $3 * 10^{7} \mathrm{~kg}$. That is, the water vapor transmitted over the preceding 24 hours far exceeded the precipitation during the precipitation event.

4.4 Mesoscale shear lines

Based on the NCEP FNL $1^{\circ} * 1^{\circ}$ analysis data (Figure 6) and RMAPS-CAv1.0 prediction (Figure 7), from 18:00 on June 25 to 00:00 on June 26, in the Hotan region of Xinjiang, the western air flow caused by the low-pressure vortex to the south of Lake Baikal 
and the Iranian high together with the eastern air flow of the weak high in the Hetao region formed a double mesoscale vortex. The horizontal scale shows a mesoscale process on the order of a $\beta$ scale.
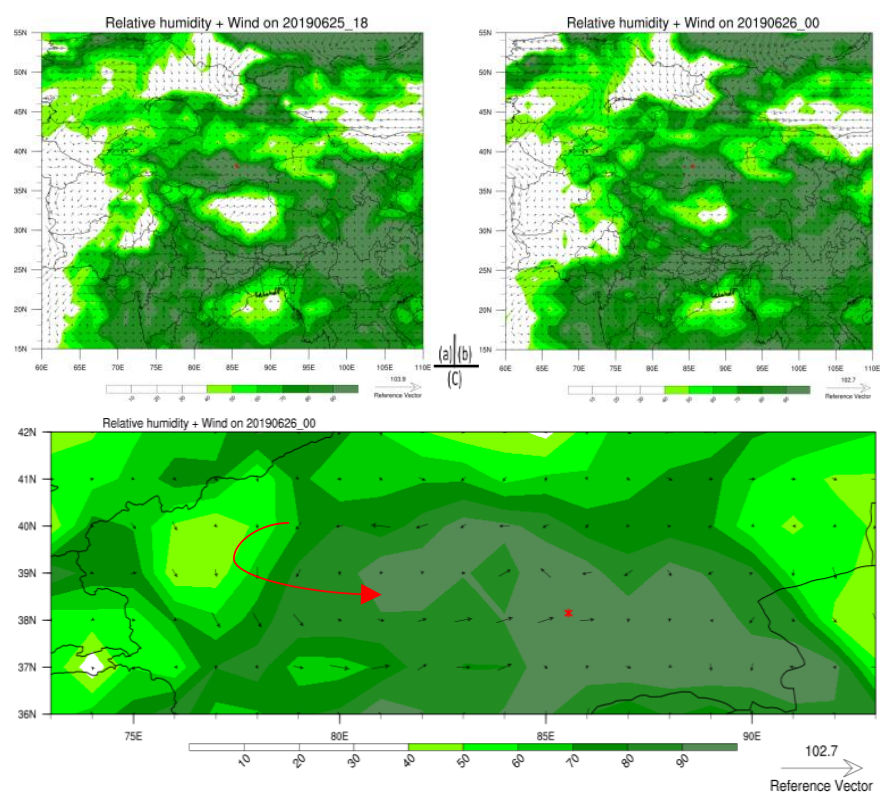

Figure 6 FNL analysis data wind vector and humidity field: a: forecast on 2019062518, b: forecast on 2019062600, c: $700 \mathrm{hPa}$ wind vector and humidity field on 2019062600

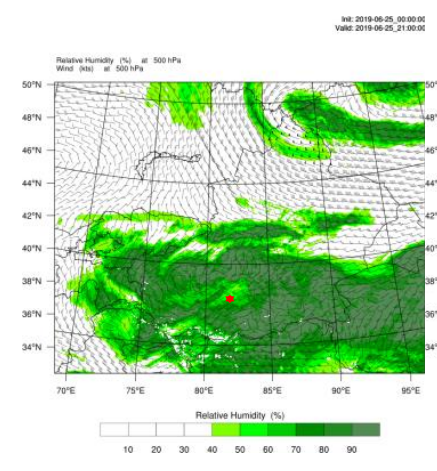

(a)

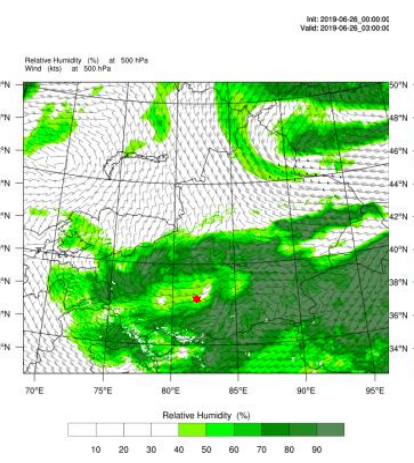

(b)

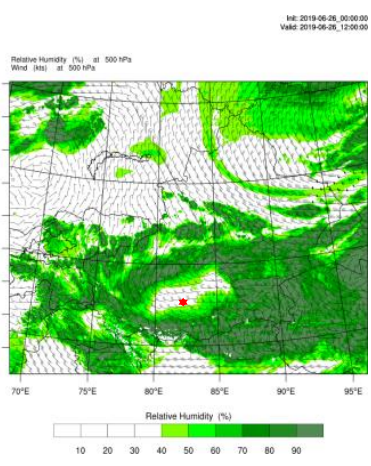

(c)

Figure 7 RMAPS-CAv1.0 wind vector and humidity forecast at $500 \mathrm{hPa}$ : (a): forecast on 2019062521, (b): forecast on 2019062603, (c)

forecast on 2019062612

\section{Conclusions}

5.1 Under the combined influence of the Iranian vortex and equatorial vortex, moisture from the 
Arabian Sea at heights above $500 \mathrm{hPa}$ can directly cross the Indus River plain traverse the Qinghai-Tibet Plateau and reach the southern region of Xinjiang. The southern transmission channel of water vapor is a very important source for the southern region of Xinjiang.

5.2 Under the cooperation in favorable terrain, the southward movement of the western Siberian cold vortex and the easterly flow of the Iranian high converged in southern Xinjiang to form a mesoscale vortex. This can cause local heavy rainfall and secondary mountain flood disasters.

5.3 Local abundant water vapor is a favorable condition for such extreme heavy precipitation. While southern Xinjiang is located in an arid portion of the continent, the frequency of local heavy rainfall has increased in recent years, which is worth further study.

Author Contributions: Investigation: D.B., Z.Z., M.E.; writing and editing: D.B., Z.Z., M.E. All authors have read and agreed to the published version of the manuscript.

Funding: The research was supported by the National Key Research and Development Program of China (2018YFC1507105).

Acknowledgments: We are grateful to S.Y. Fan for advice on this investigation.

Conflicts of Interest: The authors declare no conflict of interest.

\section{References}

Zhang Junlan, Wei Rongqing, Yang liu.A comparative analysis of the rainfall area and intensity of two rare rainstorms in Xinjiang in 2013 [J].Desert and oasis meteorology, August 2014.8 (5) :1-9.

2. Shi Yuguang,Sun zhaobo.Climatic haracteristics and changes of water vapor transport in Xinjiang [J]. Plateau meteorology, 2008,27 (2) : 310-318.

3. Shi Yuguang, Sun zhaobo, Yang qing. Areal rainfall distribution in Xinjiang [J]. Acta applied meteorology, 2008,19 (3) : 326-332.

4. Zeng Yong, Zhou Yushu, Yang Lianmei. Preliminary analysis of numerical simulation on the formation mechanism of a heavy rain in western Xinjiang [J]. Atmospheric science. 2019 (02)

5. Zeng Yong, Yang Lianmei. Cause analysis of an Extreme Rainstorm in western Xinjiang [J]. Plateau Meteorology. 2018 (05)

6. Zeng Yong, Yang Lianmei. Comparative analysis of Mesoscale Characteristics of two short-term heavy rainfall events in the west of Southern Xinjiang [J]. Rainstorm disaster. 2017 (05)

7. Zeng Yong, Yang Lianmei, Zhang Yingxin. Simulation of water vapor transport trajectory during a heavy rain in western Xinjiang [J]. Desert and Oasis Meteorology. 2017 (03) 
8. Zeng Yong, Yang Lianmei. Analysis of Mesoscale Characteristics of a rainstorm and strong convection process in the west of Southern Xinjiang [J]. Drought meteorology. 2017 (03)

9. Li Guoping, Chen Jia. New progress of Southwest Vortex and rainstorm research [J]. Rainstorm disaster. $2018(04)$

10. Li Ruqi, sun Mingjing, Li Yubo, Li Jiangang, Tang Ye. Analysis of dynamic and thermal characteristics of Rainstorm in the west of South Xinjiang [J]. Desert and Oasis Meteorology. 2017 (02)

11. Liu Rui, Yang qing. Calculation and analysis of atmospheric water vapor flux and its net income in Xinjiang [J].China desert, 2010,305 (: 1221-1228).

12. Kong Qi, Zheng Yongguang, Chen Chunyan. Synoptic-scale characteristics of the rainstorm "7.17" in urumqi [J]. Acta applicativologica sinica, 2011,22 (1) : 12-22.

Wang Minzhong, wei wenshou, Yang lianmei, et al. Process diagnosis and analysis of "7.17" heavy rainfall in Xinjiang in 2007 [J]. China desert, 2011,31 (1) : 199-206.

Li Xia, Tang hao, Yang lianmei, et al. Variability of airborne water vapor in summer in tarim basin, 1961-2000 [J]. Desert and oasis meteorology, 2011,5 (1) : 6-11.

Yang Lianmei, Zhang yunhui, Tang Hao. Characteristics of water vapor during three rainstorms in Xinjiang in July 2007 [J].Plateau meteorology, 2013,31 (4) : 963-973.

Yang liu, Yang lianmei, Tang Hao, et al. Water vapor transport characteristics in tianshan mountains from 2000 to 2011 [J]. Desert and oasis meteorology, 2013,7 (3) : 21-25.

Huoqing Li, Hailiang Zhang, Ali Mamtimin,etc al. A New Land-Use Dataset for the Weather Research and Forecasting (WRF) Model. Atmosphere 2020, 11, 350:1-19. 